\title{
Magnolol-Induced Apoptosis Is Mediated via the Intrinsic Pathway with Release of AIF from Mitochondria in U937 Cells
}

\author{
Takamichi IKaI, ${ }^{a}$ Yukihiro AKaO, ${ }^{*}, a$ Yoshihito Nakagawa, ${ }^{a}$ Kenji OHGUCHI, ${ }^{a}$ Yoshimichi SAKaI,${ }^{b}$ and \\ Yoshinori NozAWA $^{a}$ \\ ${ }^{a}$ Gifu International Institute of Biotechnology; Kakamigahara, Gifu 504-0838, Japan: and ${ }^{b}$ Gifu Prefectural Research \\ Institute of Forests; Mino, Gifu 501-3714, Japan. \\ Received June 29, 2006; accepted September 19, 2006; published online September 21, 2006
}

\begin{abstract}
Magnolol has been reported to have an inhibitory effect on tumor invasion in vitro and in vivo. In this study, we found that treatment with $30 \mu_{\mathrm{M}}$ magnolol exhibited growth inhibition partly by inducing apoptosis in cultured human leukemia U937 cells and that the apoptosis was induced via the sequential ordering of molecular events; 1) a transient decrease of phosphorylated extracelluar signal-requlated kinase (ERK), 2) translocation of apoptosis inducing factor (AIF) from mitochondria to cytosol concurrent with a decreased membrane potential, and 3) downregulation of bcl-2 protein. Pretreatment of the cells with a pan-caspase inhibitor Z-Val-Ala-Asp-fluoromethyl ketone (Z-VAD-FMK) did not prevent the apoptosis induced by magnolol. These findings indicated that the above-mentioned sequence of intracellular signaling events led to apoptosis in magnolol-treated U937 cells, which was caspase-independent.
\end{abstract}

Key words magnolol; caspase-independent apoptosis; apoptosis inducing factor (AIF); Bcl-2 family

Increasing attention has been paid to primitive medicinal plants and dietary factors to search for new substances with potentially effective anticancer activity. A large number of natural products have been evaluated as potential chemopreventive or therapeutic agents. In fact, among these compounds, paclitaxel, etoposide, camptothecin, and vincristine, have been used as anticancer drugs. Epidemiological studies have shown that dietary phytochemicals provide beneficial effects on cancer prevention. In this context, evidence-based biofactors for cancer prevention are strongly required for practical use. Among them, polyphenols are of great interest as chemopreventive agents because of their antioxidative and possible anticancer activity. ${ }^{1-4)}$

In our series of investigations to search for anticancer agents from plant sources, all the polyphenols and terpenoids tested were observed to induce apoptosis by targeting mitochondria with a decreased membrane potential, leading to the activation of the intrinsic apoptotic signal transduction. ${ }^{5-8)}$ In some cases, the early responsive signaling cascades including protein kinases mitogen-activated protein kinase (MAPK) and Akt referring to growth and survival, respectively, were down-regulated. ${ }^{6,8)}$

In previous reports, magnolol, a hydroxylated biphenyl compound isolated from Magnolia obovata has been shown to exert inhibitory effect on tumor invasion in vitro ${ }^{9}$ and in vivo. ${ }^{10)}$ In the current study, we have investigated the mechanisms underlying anti-proliferative effect of magnolol in U937 cells and have found that magnolol induced apoptosis through the intrinsic apoptotic signaling accompanied with the release of apoptosis inducing factor (AIF) from mitochondria, but not caspase activation.

\section{MATERIALS AND METHODS}

Reagents Magnolol was isolated from the stem bark of Magnolia obovata. ${ }^{9}$ Magnolol was examined for inhibitory effect on the cell growth in human monocytic leukemia cell line U937. Epigallocatechin gallate (EGCG; Wako, Osaka,
Japan) and resveratrol ${ }^{5)}$ were used as reference compounds, which were prepared as $10 \mathrm{~mm}$ in DMSO and then diluted to the desired concentration, before use.

Cell Culture, Viability, DNA Ladder Formation, and Morphological Study Human monocytic leukemia cell line U937 and Epstein-Barr (EB) virus transformed L11 Bcell line which was established in our laboratory were grown in RPMI-1640 medium supplemented with $10 \%(\mathrm{v} / \mathrm{v})$ heatinactivated fetal bovine serum (Sigma, Tokyo) and $2 \mathrm{~mm} \mathrm{L-}$ glutamine under an atmosphere of $95 \%$ air and $5 \% \mathrm{CO}_{2}$ at $37^{\circ} \mathrm{C}$. The number of viable cells was determined by the trypan blue dye-exclusion assay. For evaluating $\mathrm{IC}_{50}$, the cells were treated with magnolol at various concentrations for $72 \mathrm{~h}$. The starting cell number was $1 \times 10^{5} / \mathrm{ml}$. For evaluating apoptotic cell death, cells were seeded at a density of $1 \times 10^{5} / \mathrm{ml}$ in $15-\mathrm{mm}$ diameter wells and cultured for $24 \mathrm{~h}$, and then DNA ladder formation was examined at various times after the start of treatment with magnolol. For this, cellular DNA was extracted from whole cells by the procedure described previously. $\left.{ }^{5}\right)$ RNase was added to the DNA solution at the final concentration of $20 \mu \mathrm{g} / \mathrm{ml}$, and the mixture was incubated at $37^{\circ} \mathrm{C}$ for $30 \mathrm{~min}$. After electrophoresis on a $2.5 \%$ agarose gel, DNA was visualized by ethidium bromide staining. For morphological examination of apoptotic changes, cells were stained with Hoechst $33342(5 \mu \mathrm{g} / \mathrm{ml})$ at $37^{\circ} \mathrm{C}$ for $30 \mathrm{~min}$, washed twice with phosphate-buffered saline (PBS), pipetted dropwise onto a glass slide, and examined by fluorescence microscopy using an Olympus microscope (Tokyo, Japan) equipped with an epi-illuminator and appropriate filters. To examine the involvement of caspases in apoptosis, the tripeptide pan-caspase inhibitor Z-Val-AlaAsp-fluoromethyl ketone (Z-VAD-FMK) (MBL, Nagoya) was added $12 \mathrm{~h}$ before treatment with magnolol.

Western Blot Analysis Before and after treatment with magnolol, U937 cells were washed twice with PBS, lysed in lysis buffer $\mathrm{A}, \mathrm{B}$ or $\mathrm{C}$, and then homogenized. Lysis buffer A $(2 \times \mathrm{PBS}, 0.1 \%$ SDS, $1 \%$ Nonidet P- $40,0.5 \%$ sodium deoxycholate and $25 \times$ Complete, protease inhibitor (Roche, 
Penzberg Germany)) was used to analyze caspase-3, -8, -9, $\mathrm{Bad}$, Bax and Bcl-2. Lysis buffer B (250 mm sucrose, $20 \mathrm{~mm}$ Hepes-KOH (pH 7.5), $10 \mathrm{~mm} \mathrm{KCl}, 1.5 \mathrm{~mm} \mathrm{MgCl}_{2}, 1 \mathrm{~mm}$ EDTA, $1 \mathrm{~mm}$ EGTA, $1 \mathrm{~mm}$ DTT and $25 \times$ Complete) was used to analyze AIF, cytochrome $c$ and endonuclease G. Lysis buffer $\mathrm{C}(250 \mathrm{~mm}$ sucrose, $20 \mathrm{~mm}$ Hepes-KOH (pH 7.5), $10 \mathrm{~mm} \mathrm{KCl}, 1.5 \mathrm{~mm} \mathrm{MgCl}_{2}, 1 \mathrm{~mm}$ EDTA, $1 \mathrm{~mm}$ EGTA, $1 \mathrm{~mm}$ DTT, $1 \%$ Nonidet P-40, $25 \times$ Complete, and Posphatase Inhibitor Cocktail 1 and 2 (Sigma-Aldrich Co.)) was used to analyze mitogen activated protein (MAP) kinases and Akt kinase. The mitochondrial and cytosolic fractions were prepared as reported previously. ${ }^{5}$ ) Ten micrograms of protein of each cell lysate was separated by SDS-PAGE by using and electroblotted onto a PVDF membrane (Du Pont, Boston, MA, U.S.A.). After blockage of nonspecific binding sites for $1 \mathrm{~h}$ by $5 \%$ nonfat milk in TPBS (PBS and $0.1 \%$ Tween 20 ), the membrane was incubated overnight at $4{ }^{\circ} \mathrm{C}$ with various human antibodies. They include anti-caspase-3 (Santa Cruz Biotechnology, CA, U.S.A.), anti-caspase-8 (MBL, Nagoya, Japan), anti-caspase-9 (Novus Biologicals Inc., CO, U.S.A.), anti-Bad (Cell Signaling Technology Inc., MA, U.S.A.) antiAIF (ProSci Inc., CA, U.S.A.), anti-cytochrome $c$ (Upstate Biotechnology Inc., NY, U.S.A.), anti-endonuclease G (Sigma-Aldrich Co.), anti-p44/42 ERK (Cell Signaling Technology Inc., MA, U.S.A.), anti-phospho-p44/42 extracelluar signal-regulated kinase (ERK) (Thr202/Tyr204) (Cell Signaling Technology Inc.), anti-SAPK/JNK (JNK) (Cell Signaling Technology Inc.), anti-phospho-SAPK/JNK (Thr183/Tyr185) (p-JNK) (Cell Signaling Technology Inc.), anti-p38 MAP kinase (p38) (Cell Signaling Technology Inc.), anti-phosphop38 MAP kinase (Thr180/Tyr182) (p-p38) (Cell Signaling Technology Inc.), anti-phospho-Akt (Cell Signaling Technology Inc.), anti-Bcl-2 (Santa Cruz Biotechnology), anti-Bax

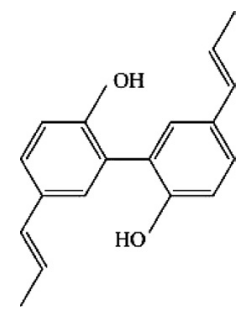

Fig. 1. Chemical Structure of Magnolol Used in This Study
(Cell Signaling Technology Inc.), and anti- $\beta$-actin (SigmaAldrich Co.). There membranes were then washed three times with TPBS, incubated further with alkaline phosphatase-conjugated goat anti-mouse antibody (Promega, Madison, WI, U.S.A.) or anti-rabbit antibody (New England Biolabs, Beverly, MA, U.S.A.) at room temperature, and then washed three times with TPBS. The immunoblot was visualized by use of an enhanced chemiluminescence detection kit (New England Biolabs).

Measurement of Mitochondrial Membrane Potential Using Mito-Traker Probe Mitochondrial membrane potential was measured by use of a fluorescent dye, MitoTracker Orange (Molecular Probes, \#M-7511) which accumulates selectively in active mitochondria and becomes to be fluorescent when oxidized. After the cells were treated with Mito-Tracker Orange solution and washed twice with PBS, the cells were resuspended in PBS. The fluorescence of Mito-Tracker Orange in the cells was observed under fluorescence microscopy (Olympus, Tokyo, Japan). ${ }^{7,8)}$

\section{RESULTS AND DISCUSSION}

Growth Inhibitory Effect of Magnolol in U937 Cells We examined the effect of magnolol (Fig. 1), a hydroxylated biphenyl compound, at various concentrations on the growth in a human leukemia cell line U937, and the results are shown in Fig. 2. Magnolol caused growth inhibition against the cells, as judged by trypan blue-exclusion test. It was noted that the susceptibility of U937 cells to magnolol was higher than that to EGCG, but was lower than that to resveratrol (Fig. 2A). The $\mathrm{IC}_{50}$ of magnolol was $27.2 \mu \mathrm{M}$. Magnolol also exhibited a growth inhibitory effect in EB virus-transformed L11 B-cells which have no genomic abnormality as well as resveratorol and EGCG (Table 1). Thus, the effect of them was most likely found not only in tumor cells but also in growing normal cells. Then we examined the mechanism

Table 1. $\mathrm{IC}_{50}$ of Magnolol in Human EB Virus-Transformed Cell Line L11

\begin{tabular}{cccc}
\hline \hline Compounds & Magnolol & Resveratrol & EGCG \\
\hline $\mathrm{IC}_{50}(\mu \mathrm{M})$ & 31.6 & 16.7 & 24.7 \\
\hline
\end{tabular}

(A)

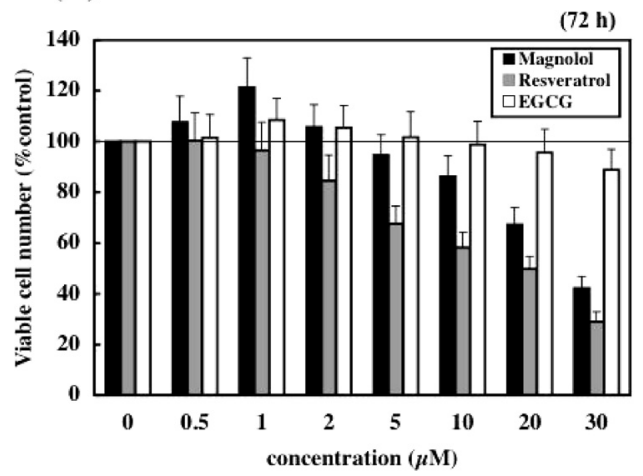

(B)

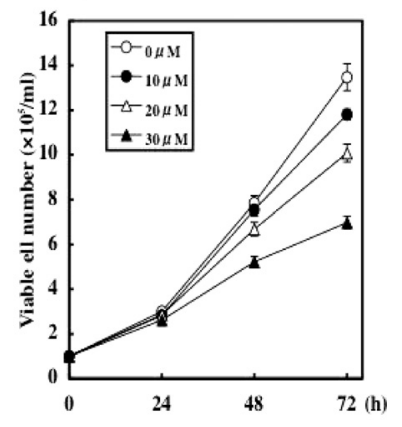

Fig. 2. Effect of Magnolol on Cell Growth in Human Leukemia U937 Cells

$1 \times 10^{5}$ cells/well were innoculated in 24 -well plate dishes, to which magnolol dissolved in DMSO or DMSO alone, were added at $24 \mathrm{~h}$ after cultivation. (A) Dose-dependent growth inhibition of magnolol, resveratorol, and EGCG. (B) Time course of viable cell numbers after treatment with various concentrations of magnolol was evaluated by the trypan-blue dye exclusion test. Each value represents the mean \pm S.D. of 3 independent experiments. 
(A)
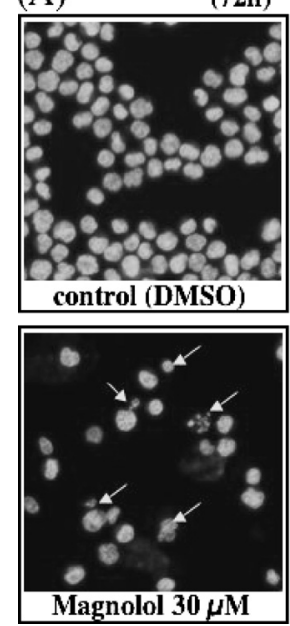

(B)

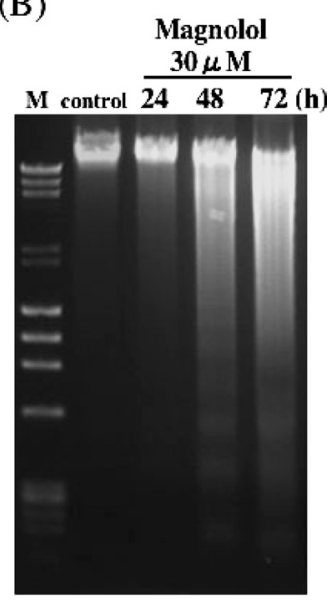

(C)

(D)
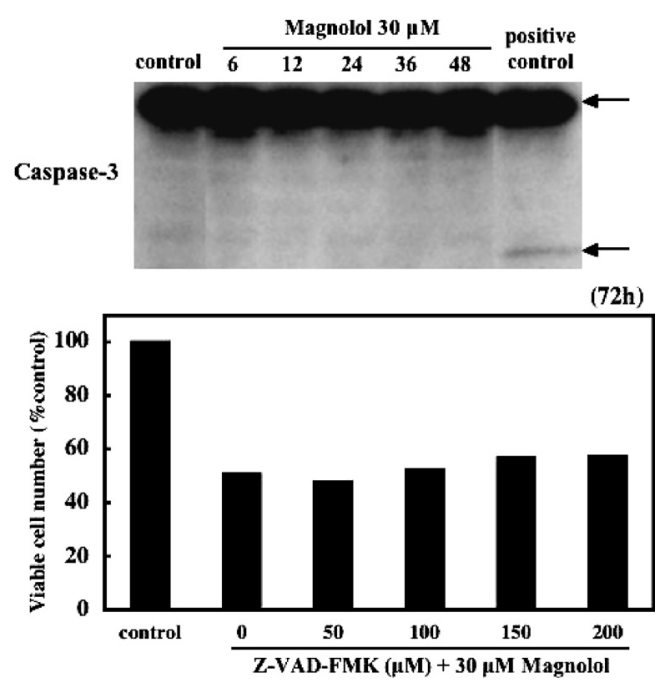

for the growth suppression in U937 cells exposed to magnolol.

The growth of the U937 cells was markedly suppressed by the magnolol treatment at more than $20 \mu \mathrm{M}$, as compared with the control (Fig. 2B). When treated with $30 \mu \mathrm{M} \mathrm{mag-}$ nolol for $72 \mathrm{~h}$, we observed apoptotic changes, which were assessed by morphological parameters (nuclear condensation and fragmentation) (Fig. 3A) and DNA ladder formation (48-72 h) in U937 cells (Fig. 3B). Taken together, it was indicated that the suppression of cell growth by magnolol was attributed mainly to apoptotic cell death.

Mechanism of Magnolol-Induced Apoptosis in U937 Cells Apoptosis has been well known to be executed by the cascade activation of initiator caspases (e.g. caspase-8, -9) and executioner ones (e.g. caspase-3, -7). To know which caspase(s) is involved in magnolol-induced apoptosis, we examined formation of active forms of caspases in cell lysate by Western blot analysis. Any processed active forms of the caspases tested were not observed in U937 cells after the treatment with $30 \mu \mathrm{m}$ magnolol. As shown in Fig. 3C, an executioner caspase- 3 was not processed. Furthermore, pretreatment with the pan-caspase-like protease inhibitor Z-VADFMK did not prevent growth inhibition by magnolol (Fig. $3 \mathrm{D})$. These findings indicate that caspase may not be in-
(E)

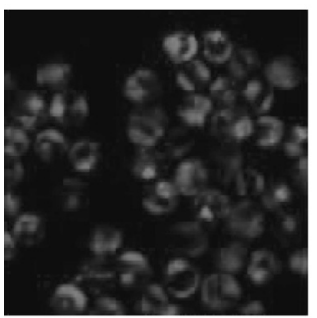

control (DMSO)
(48 h)

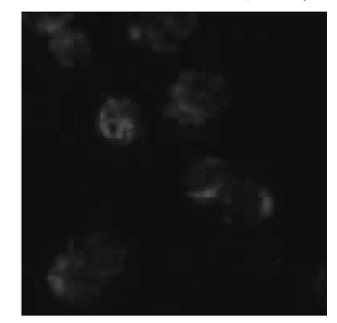

Magnolol $30 \mu \mathrm{M}$
(F)

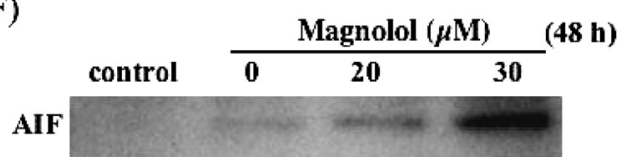

Fig. 3. Magnolol-Induced Apoptosis in U937 Cells

(A) Morphological features of U937 cells treated with $30 \mu \mathrm{M}$ magnolol for $72 \mathrm{~h}$. The cells were stained with Hoechst $33342(5 \mu \mathrm{g} / \mathrm{ml})$ for $30 \mathrm{~min}$ and then examined by fluorescence microscopy. (Upper panel) cells treated with DMSO alone (control); (Lower panel) $30 \mu \mathrm{m}$ magnolol. (B) Nucleosomal DNA fragmentation in U937 cells exposed to $30 \mu \mathrm{m}$ magnolol for different intervals. Three micrograms of DNA were loaded onto each lane. Lane M is a DNA size marker. (C) Effect on caspase-3 activation after the treatment with $30 \mu \mathrm{M}$ magnolol examined by Western blot analysis. Control is a sample from the cells prior to the treatment, and the positive control showing caspase- 3 activation is a sample from human colon cancer SW480 cells treated with arsenic trioxide $5 \mu \mathrm{M}$ for $36 \mathrm{~h}^{13)}$ (D) Effect of pan-caspase inhibitor, Z-VAD-FMK, on $30 \mu \mathrm{M}$ magnololinduced apoptosis. The inhibitor was added $12 \mathrm{~h}$ before exposure to $30 \mu \mathrm{M}$ magnolol. The rescue of cell death was evaluated at $72 \mathrm{~h}$ after the treatment with $30 \mu \mathrm{m}$ magnolol in the presence of different concentrations of the inhibitor. (Control) cells in the presence of $0.01 \%$ DMSO and $200 \mu \mathrm{M}$ Z-VAD-FMK. Results are the mean of 2 independent experiments. (E) Mitochondrial membrane potential after $48 \mathrm{~h}$-treatment with $30 \mu \mathrm{m}$ magnolol. (Left) control (DMSO alone), (Right) $30 \mu \mathrm{m}$ magnolol-treated. (F) Release of AIF at $48 \mathrm{~h}$ after the treatment with $30 \mu \mathrm{m}$ magnolol examined by Western blot analysis.

volved in the magnolol-induced apoptosis.

Next we examined the mitochondrial pathway which plays a crucial role in propagation and determination of cell death. In $48 \mathrm{~h}$-treated cells, the mitochondrial membrane potential was markedly decreased when examined by the use of MitoTracker fluorescent probe (Fig. 3E). Although, the level of Bcl-2 expression, which inhibits the loss of mitochondrial membrane potential induced by apoptotic signals, was gradually increased in time-dependent manner even in the cells without the treatment, it was decreased in $48 \mathrm{~h}$-treated cells compared with the control cells. On the other hand, the level of Bax was increased (Fig. 4A). The level of c-myc protein was also markedly reduced at that time (Fig. 4A). These findings suggested that cell growth was inhibited in the majority of the cells, in some of which apoptosis was induced at $48 \mathrm{~h}$ after the treatment. On the other hand, Western blot analysis did not show the apparent band of released cytochrome c after the magnolol treatment (data not shown). Consequently, caspase-9, which is known to bind to the cytochrome c/Apaf1 complex, was not activated after the treatment (data not shown). Therefore, we examined two other potent apoptosisinducing potent factors, AIF and endonuclease G, which were known to be released from mitochondria in the apoptotic process. Western blot analysis revealed that the amount 


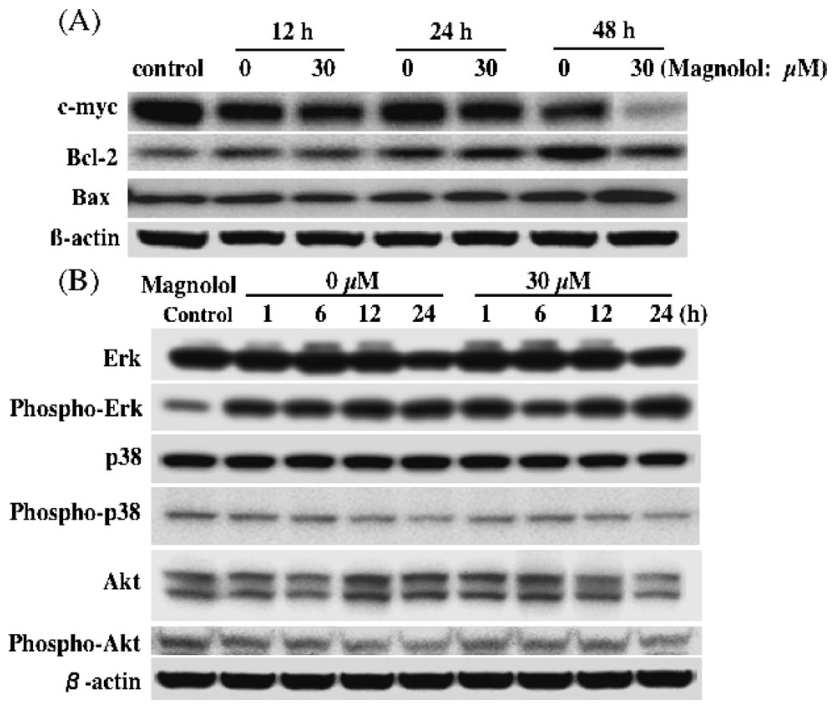

Fig. 4. Death-Associated Signaling of Magnolol-Induced Cell Death in U937 Cells

(A) The expression levels of c-myc, Bcl-2 and Bax examined by Western blot analysis. (B) The expression of p44/42 ERK, phospho-p44/42 ERK, p38 MAPK, phosphop38 MAPK, Akt kinase, and phospho-Akt kinase examined by Western blot analysis.

of released active AIF at $48 \mathrm{~h}$ after the magnolol treatment was dose-dependently increased (Fig. 3F), but endonuclease $\mathrm{G}$ was not detected (data not shown).

Effect of Magnolol on MAP Kinases and Akt Kinase We examined the activation of MAP kinases in magnolol-induced growth inhibition (Fig. 4B). Among the kinases, the level of p-p44/42 ERK (pERK) greatly increased at $1 \mathrm{~h}$ after the magnolol treatment and then transiently decreased at $6 \mathrm{~h}$ (Fig. 4B). p-JNK was nonspecifically increased at $1 \mathrm{~h}$ after the treatment (data not shown). p-p38 and p-Akt were unchanged.

Magnolol, a polyphenol from Magnolia obovata, has been proved to possess an inhibitory effect on tumor invasion in vitro ${ }^{9)}$ and in vivo. ${ }^{10)}$ In the current study, we demonstrated that magnolol induced an apoptotic cell death through the type II apoptotic signaling pathway via mitochondria without caspase-activation in human monocytic leukemia U937 cells. It has become increasingly clear that mitochondria play a major rate-limiting role in apoptosis. The decision/effecter phase of the apoptotic process converges on mitochondria, where permeabilization of mitochondrial membranes is triggered, and apoptosis inducing factors such as cytochrome c, AIF, and endonuclease $G$ are released. Our present study has shown that magnolol-induced apoptosis was mediated by a death factor AIF, as judged by Western blotting indicating that the released AIF level was increased after exposure to magnolol at either 20 or $30 \mu \mathrm{M}$. Furthermore, the mitochondrial membrane potential was decreased. Concurrently, Bcl-2 was down-regulated while Bax was up-regulated.

Although AIF is known to be a potent factor in the caspase-independent apoptosis, it is not clear as to how AIF causes DNA ladder formation. It has been reported that released AIF was localized around nuclei and partly translo- cated into nuclei after the treatment of the apoptogenic dolichyl monoposphate in U937 cells, and that both caspase3 and -8 inhibitors blocked only DNA fragmentation but not AIF migration and chromatin condensation. ${ }^{11)}$ In our study, the pan-caspase inhibitor did not prevent the apoptotic cell death induced by magnolol. Accordingly, our data raise the possibility that AIF, but not caspases, may play a pivotal role in DNA ladder formation, as reported previously in arucanolide-induced apoptosis. ${ }^{8)}$

It should be, moreover, noted that magnolol significantly increased the expression of Bax protein, whereas it decreased Bcl-2 expression. Yang et al. also reported that magnolol modulates the expression of Bcl-2 family in $\mathrm{CH} 27$ human lung squamous carcinoma cells. ${ }^{12}$ ) These results altogether indicated that the release of AIF with a decreased membrane potential and also modulation of the expression of Bcl-2 protein family may be responsible for magnolol-induced apoptosis in U937 cells. The polyphenols and terpenoids exhibiting an anti-cancer activity have been shown to affect activation of MAPK and Akt pathways. ${ }^{6,8)}$ In magnolol-induced apoptosis, the increased level of pERK $1 / 2$ at $1 \mathrm{~h}$ after treatment was transiently reduced at $6 \mathrm{~h}$ after the treatment, but, phosphorylation levels of p38 and JNK kinases were not altered. Recently, it was reported that ERK $1 / 2$ in glutamate toxicity in HT22 mouse hippocampal cells plays dual roles in acting as part of a cellular adaptive response during the initial phases of glutamate-induced oxidative stress and contributing to toxicity during later stages of stress. ${ }^{13)}$ Such dual responses were appeared in the current case. Further study to clarify the relationship between the early response signal and the apoptosis signal will be needed. Nevertheless, magnolol, which is constituent of Chinese medicinal herb, could be a potentially effective candidate for chemoprevention.

\section{REFERENCES}

1) Nabekura T, Kamiyama S, Kitagawa S., Biochem. Biophys. Res. Commun., 327, 866-870 (2005)

2) Greenwald P., Recent Results Cancer Res., 166, 1-15 (2005).

3) Lee K. W., Lee H. J., Lee C. Y., Crit. Rev. Food Sci. Nutr., 44, 437452 (2004).

4) Liu R. H., J. Nutr., 134, 3479S-3485S (2004).

5) Ito T., Akao Y., Yi H., Ohguchi K., Matsumoto K., Tanaka T., Inuma M., Nozawa Y., Carcinogenesis, 24, 1489-1497 (2003).

6) Ohguchi K., Akao Y., Matsumoto K., Tanaka T., Ito T., Iinuma M., Nozawa Y., Biosci. Biotechnol. Biochem., 69, 353-356 (2005).

7) Matsumoto K., Akao Y., Yi H., Ohguchi K., Ito T., Tanaka T., Kobayashi E., Iinuma M., Nozawa Y., Bioorg. Med. Chem., 12, 5799-5806 (2004).

8) Nakagawa Y., Iinuma M., Matsuura N., Yi K., Naoi M., Nakayama T., Nozawa Y., Akao Y., J. Pharmacol. Sci., 97, 242-252 (2005).

9) Nagase H., Ikeda K., Sakai Y., Planta Med., 67, 705-708 (2001).

10) Ikeda K., Sakai Y., Nagase H., Phytother. Res., 17, 933-937 (2003).

11) Yasugi E., Kumagai T., Nishikawa Y., Okuma E., Saeki K., Oshima M., Susin S. A., Kroemer G., Yuo A., FEBS Lett., 480, 197-200 (2000).

12) Yang S. E., Hsieh M. T., Tsai T. H., Hsu S. L., Br. J. Pharmacol., 138, 193-201 (2003).

13) Luo Y., DeFranco D. B., J. Biol. Chem., 281, 16436-16442 (2006). 